\title{
Diagnóstico precoce de dissecção aórtica em pacientes com dor torácica: uma revisão integrativa da literatura
}

\author{
Early diagnosis of aortic dissection in patients with chest pain: an integrative literature review \\ Diagnóstico precoz de disección aórtica en pacientes con dolor torácico: una revisión integradora \\ de la literatura
}

Recebido: 01/02/2021 | Revisado: 05/02/2021 | Aceito: 08/02/2021 | Publicado: 20/02/2021

Olívio Joaquim Fonseca Neto ORCID: https://orcid.org/0000-0002-2695-0077 Universidade Federal do Delta do Parnaíba, Brasil

E-mail: oliviofonsecaneto@hotmail.com

Isabella Pires Gomes Mendes

ORCID: https://orcid.org/0000-0003-3486-7908

Universidade Federal do Delta do Parnaíba, Brasil

E-mail: isabellapiresmendes@gmail.com

Andressa Carvalho Pereira

ORCID: https://orcid.org/0000-0001-7721-0661

Universidade Federal do Delta do Parnaíba, Brasil

E-mail: andressa_carvalho@ufpi.edu.br

Juliano Luiz de Souza

ORCID: https://orcid.org/0000-0003-0567-2167

Universidade Federal do Delta do Parnaíba, Brasil

E-mail: julianoluizdesouza@ufpi.edu.br

Brenda Larissa Andrade Viana

ORCID: https://orcid.org/0000-0003-2285-4309

Universidade Federal do Delta do Parnaíba, Brasil

E-mail: brendalarissa.av@gmail.com

Yasser da Silveira Krüger

ORCID: https://orcid.org/0000-0002-6163-7553

Universidade Federal do Delta do Parnaíba, Brasil

E-mail: yasser.kruger@gmail.com

João Victor Carvalho Barbosa

ORCID: https://orcid.org/0000-0001-7790-0312

Universidade Federal do Delta do Parnaíba, Brasil

E-mail: joaovictorcarvalhob@gmail.com

Cláudio Vinícius Barroso Queirós de Lima

ORCID: https://orcid.org/0000-0002-4047-8637

Universidade Federal do Delta do Parnaíba, Brasil

E-mail: cld.vinicius99@gmail.com

Priscylla Frazão Rodrigues

ORCID: https://orcid.org/0000-0001-7580-516X

Universidade Federal do Delta do Parnaíba, Brasil

E-mail: priscyllafrazao1995@gmail.com

Adrielly Cristhine Gonçalves Araujo

ORCID: https://orcid.org/0000-0002-2925-2231

Universidade Federal do Delta do Parnaíba, Brasil E-mail: adriellygoncalves@ufpi.edu.br

João Pedro Sousa Mendes

ORCID: https://orcid.org/0000-0002-0721-2845

Universidade Federal do Delta do Parnaíba, Brasil E-mail: mendespedro00@gmail.com

Igor dos Santos Cavalcante

ORCID: https://orcid.org/0000-0003-2907-9026

Universidade Federal do Delta do Parnaíba, Brasil E-mail: igorsc@live.com

Antonino Neto Coelho Moita

ORCID: https://orcid.org/0000-0002-4993-2568

Universidade Federal do Delta do Parnaíba, Brasil

E-mail: antoninomoita@ufpi.edu.br

Elias de Carvalho Magalhães Neto

ORCID: https://orcid.org/0000-0002-7494-7147

Universidade Federal do Delta do Parnaíba, Brasil E-mail: elias.nt@hotmail.com 


\begin{abstract}
Resumo
Introdução: A Dissecção Aórtica Aguda (DAA) é definida como uma súbita ruptura da camada íntima da aorta, expondo a camada subjacente ao fluxo sanguíneo e criando um "falso lúmen" paralelo à parede aórtica. A DAA é um agravo extremamente letal e que requer rápida identificação para iniciar o tratamento cedo e evitar a morte do paciente. Esta revisão teve como objetivo descrever as principais manifestações clínicas da DAA e apresentar estratégias para a identificação precoce e eficiente desse agravo no departamento de emergência. Métodos: Foi realizada uma revisão integrativa de estudos publicados nas plataformas PubMed e Google Scholar com os descritores: "aortic dissection" AND “chest pain". Foram selecionados 37 artigos, 12 foram excluídos e 25 estudos foram utilizados na revisão. Resultados e Discussão: Para diagnóstico precoce, além de análise de sinais e sintomas, podem ser úteis na investigação exames complementares como radiografia, tomografia computadorizada, ressonância magnética, ecocardiografia, eletrocardiograma, ultrassonografia cardíaca focalizada (FOCUS) e exame de sangue incluindo enzimas cardíacas. Conclusão: Os estudos analisados possibilitaram o entendimento patológico da DAA, bem como a apresentação de formas eficientes de diagnóstico e de tratamentos do agravo.
\end{abstract}

Palavras-chave: Dissecção aórtica; Dor torácica; Diagnóstico.

\begin{abstract}
Introduction: Acute Aortic Dissection (AAD) is defined as a sudden rupture of the intimate layer of the aorta, exposing the underlying layer to blood flow and creating a "false lumen" parallel to the aortic wall. DAA is an extremely lethal condition that requires rapid identification to start treatment early and prevent patient death. This review aimed to describe the main clinical manifestations of $\mathrm{AAD}$ and present strategies for the early and efficient identification of this condition in the emergency department. Methods: An integrative review of studies published on the PubMed and Google Scholar platforms was performed with the descriptors: "aortic dissection" AND "chest pain". 37 articles were selected, 12 were excluded and 25 studies were used in the review. Results and Discussion: For early diagnosis, in addition to analyzing signs and symptoms, complementary tests such as radiography, computed tomography, magnetic resonance imaging, echocardiography, electrocardiogram, focused cardiac ultrasound (FOCUS) and blood tests including cardiac enzymes may be useful in the investigation. Conclusion: The studies analyzed enabled the pathological understanding of AAD, as well as the presentation of efficient forms of diagnosis and treatment of the disease.
\end{abstract}

Keywords: Aortic dissection; Chest pain; Diagnosis.

\title{
Resumen
}

Introducción: La disección aórtica aguda (DAA) se define como una ruptura repentina de la capa íntima de la aorta, exponiendo la capa subyacente al flujo sanguíneo y creando una "falsa luz" paralela a la pared aórtica. La DAA es una enfermedad extremadamente letal que requiere una identificación rápida para iniciar el tratamiento temprano y prevenir la muerte del paciente. Esta revisión tuvo como objetivo describir las principales manifestaciones clínicas de la DAA y presentar estrategias para la identificación temprana y eficiente de esta condición en el servicio de urgencias. Métodos: Se realizó una revisión integradora de estudios publicados en las plataformas PubMed y Google Scholar con los descriptores: "aortic dissection" AND "chest pain". Se seleccionaron 37 artículos, 12 fueron excluidos y se utilizaron 25 estudios en la revisión. Resultados y discusión: Para el diagnóstico temprano, además del análisis de signos y síntomas, las pruebas complementarias como radiografía, tomografía computarizada, resonancia magnética, ecocardiografía, electrocardiograma, ultrasonido cardíaco enfocado (FOCUS) y análisis de sangre que incluyen enzimas cardíacas pueden ser útiles en la investigación. Conclusión: Los estudios analizados permitieron la comprensión patológica de la DAA, así como la presentación de formas eficientes de diagnóstico y tratamiento de la enfermedad.

Palabras clave: Disección aórtica; Dolor torácico; Diagnóstico.

\section{Introdução}

As doenças cardiovasculares representam a principal causa de morte em todo mundo (Chang et al., 2017). Nesse contexto, as doenças da aorta se apresentam como importantes causas de morbimortalidade no departamento de emergência (DE) e como um desafio permanente a cardiologistas e cirurgiões (Albuquerque et al., 2009).

Define-se como Dissecção Aórtica Aguda (DAA) o evento em que há uma súbita ruptura da camada íntima da artéria, expondo a camada subjacente ao fluxo sanguíneo e, portanto, criando um "falso lúmen" paralelo à parede aórtica. Um mecanismo menos comum é caracterizado pelo sangramento dentro da parede do vaso, referido como hematoma intramural aórtico. A progressão da dissecção pode causar grave hemorragia e levar à morte rapidamente (Gawinecka et al., 2017; Elsayed et al., 2017). Possíveis complicações da DAA incluem síndrome de má perfusão letal, regurgitação aórtica, tamponamento cardíaco, infarto agudo do miocárdio e acidente vascular cerebral (Gawinecka et al., 2017). 
O comprometimento da parede aórtica - seja por doenças congênitas do tecido conjuntivo ou por condições adquiridas, como a aterosclerose - é o principal fator etiopatogênico associado à ocorrência de dissecção. A hipertensão arterial é um dos principais fatores de risco para esse agravo e está presente em até 75\% dos casos (Gawinecka et al., 2017; Nienaber \& Clough, 2015). Outros fatores de risco incluem tabagismo, traumatismo contuso direto e uso de drogas, como a cocaína e as anfetaminas (Nienaber \& Clough, 2015).

A classificação da dissecção aórtica baseia-se principalmente na localização anatômica e no tempo de evolução (Nienaber \& Clough, 2015). O sistema de classificação DeBakey diferencia as dissecções em tipo I (acomete a aorta ascendente e progride para os demais segmentos aórticos), tipo II (fica confinada à aorta ascendente) e tipo III (envolve apenas a aorta descendente) (Gawinecka et al., 2017). Já de acordo com o sistema de classificação de Stanford, mais comumente utilizado, dissecções envolvendo a aorta ascendente são classificadas como tipo A, enquanto aquelas que não envolvem esse segmento são classificadas como tipo B (Elsayed et al., 2017; (Nienaber \& Clough, 2015). Com relação ao tempo de início dos sintomas, a dissecção aórtica pode ser descrita como hiperaguda (<24 horas), aguda (2-7 dias), subaguda (8-30 dias) e crônica ( $>30$ dias) (Nienaber \& Clough, 2015).

A DAA tem mortalidade de $1 \%$ a $2 \%$ por hora após o início dos sintomas em pacientes não tratados (Asha \& Miers, 2015). Ademais, a morte após um curto período de sintomas ocorre em uma quantidade significativa de pacientes (Elsayed et al., 2017). A rápida identificação desse agravo é fundamental para diminuir a taxa de mortalidade. No entanto, o diagnóstico da DAA ainda é desafiador, pois os sinais e sintomas são inespecíficos e a maioria dos diagnósticos diferenciais é bem mais frequente do que a DAA (Nazerian, Vanni, et al., 2014). Por ser um agravo extremamente letal, a DAA deve ocupar uma posição importante na suspeita diagnóstica em pacientes com dor torácica aguda que chegam ao DE (Gawinecka et al., 2017).

As diretrizes clínicas recomendam que a DAA seja considerada em todos os pacientes que apresentem dor torácica, dor nas costas, dor abdominal, síncope ou sintomas consistentes com déficit de perfusão (Nazerian, Giachino, et al., 2014). O escore de risco de detecção aórtica, proposto pelas diretrizes da American Heart Association e do American College of Cardiology, é uma ferramenta simples de triagem à beira do leito que pode ser utilizado para estratificar os pacientes em probabilidade baixa, intermediária e alta de DAA (Nazerian, Vanni, et al., 2014). Entretanto, outras estratégias diagnósticas ainda são necessárias para auxiliar na avaliação inicial desses pacientes e para aumentar a taxa de detecção da DAA (Nazerian, Giachino, et al., 2014).

O objetivo desse artigo de revisão é descrever as principais manifestações clínicas da DAA e apresentar estratégias para a identificação precoce e eficiente desse agravo no departamento de emergência.

\section{Metodologia}

O presente artigo trata-se de um estudo de natureza qualitativa baseado no método de revisão integrativa da literatura, descrito por Pereira et al. (2018), no qual é relevante usar apenas autores centrados no tema abordado, para que, assim, possam ser usados também na discussão. Para sua elaboração foram obedecidas as normas pertinentes de amostragem na literatura, coleta de dados, análise crítica dos estudos incluídos e discussão dos resultados. Para isso, foi selecionado um tema e definido o objetivo de pesquisa. Em seguida, foram estabelecidos os fatores de inclusão e de exclusão.

Dessa forma, foi estabelecido o tema "dissecção aórtica em pacientes com dor torácica" e objetivou-se pesquisar e apresentar as melhores formas possíveis de garantir um diagnóstico precoce e preciso de dissecção aórtica em um paciente da emergência que apresenta dor torácica, visando ajudar acadêmicos e profissionais a garantir um bom prognóstico.

Para a pesquisa bibliográfica realizada em outubro de 2020 foram utilizadas as bases de dados PubMed e Google Scholar. Para a busca de artigos na primeira plataforma foram utilizados os termos de busca "aortic dissection" e "chest pain" com o auxílio do operador booleano “AND”. De modo a seguir os critérios de inclusão estabelecidos, foram marcadas todas as 
seguintes modalidades de artigos: "Books and Documents", "Clinical Trial", "Meta-Analysis", "Randomized Controlled Trial" e "Systematic Review". Também foi aplicado o filtro de textos que estivessem disponíveis de forma integral, gerando assim, seis (6) artigos como resultado.

Em segundo momento, para a busca de artigos na base de dados Google Scholar, foi utilizada a ferramenta computacional Publish or Perish, utilizando-se novamente os termos "aortic dissection" e "chest pain" e o operador booleano “AND” e incluindo artigos publicados entre 2014 e 2020. Com a utilização da ferramenta Publish or Perish, foram incluídos no resultado apenas artigos que atendessem o índice $\mathrm{H}$ de qualidade, gerando como resultado de busca trinta e um (31) artigos.

Com um total de trinta e sete (37) artigos analisados, dez (10) foram excluídos após leitura do título de do resumo. Em seguida, os vinte e sete (27) artigos restantes foram submetidos à leitura integral, de forma que dois (2), por fim, foram excluídos.

Os critérios de inclusão utilizados foram artigos científicos escritos em inglês ou em português, disponibilizados integralmente, que fossem publicados entre o período de 2014 a 2020 e que apresentassem acompanhamento de pacientes com dor torácica acometidos por dissecção aórtica, selecionando apenas os artigos de maior relevância. Quanto aos critérios de exclusão, foram desconsiderados materiais que não fossem artigos científicos, que não estivem dentro do período de 2014 a 2020, artigos que fossem revisões não sistemáticas da literatura e estudos que não se relacionavam ao tema selecionado. (Figura 1)

Figura 1 - Fluxograma detalhando as etapas do processo de seleção de artigos para revisão.

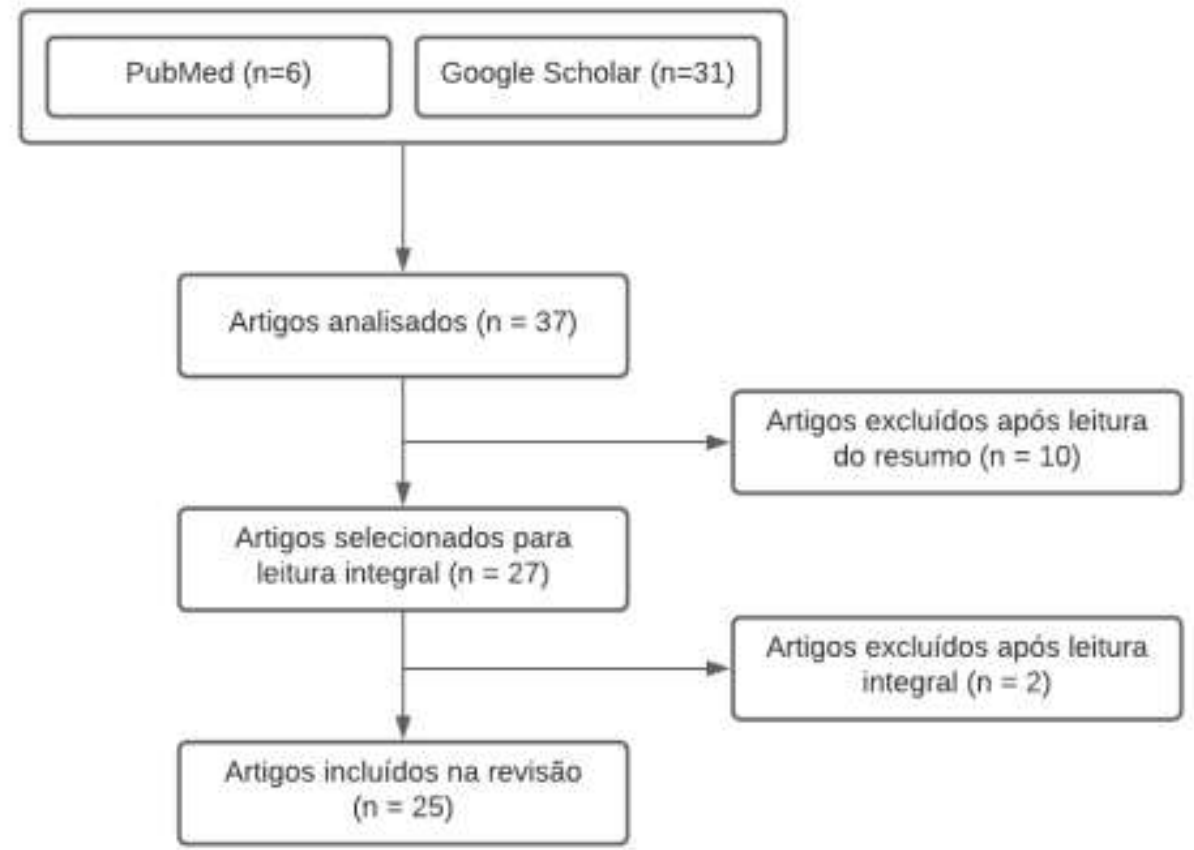

Fonte: Autores.

\section{Resultados e Discussão}

\section{Identificação e diagnóstico precoce}

A DAA apresenta um quadro clínico de sinais e sintomas inespecífico, sendo comumente confundida com outras causas de dor torácica mais frequentes como eventos isquêmicos do miocárdio e tromboembolismo pulmonar, o que torna seu diagnóstico, na maioria dos casos, difícil e desafiador (Hsia et al., 2016; Gawinecka et al., 2017; Pape et al., 2015). Nesse cenário, o diagnóstico da DAA, por muito tempo, era feito apenas post-mortem e, até mesmo atualmente, com a evolução das modalidades 
diagnósticas e, consequentemente, melhoria dos resultados nas últimas 4 décadas, até 30\% dos pacientes tem seu diagnóstico inicial incorreto (Gawinecka et al., 2017; Elsayed et al., 2017). Assim, a letalidade dessa emergência continua alta, sendo de aproximadamente $1 \%$ por hora após o início dos sintomas em pacientes que não recebem um manejo inicial adequado (Gawinecka et al., 2017; Elsayed et al., 2017).

Desse modo, é preciso um alto índice de suspeita clínica para que seja feito um diagnóstico exato precocemente (Gawinecka et al., 2017; Elsayed et al., 2017). Para isso, uma boa análise clínica, composta por uma anamnese detalhada e um exame físico completo, é uma ferramenta essencial para o diagnóstico de DAA (Elsayed et al., 2017; Pape et al., 2015). Segundo (Pape et al., 2015), apesar de alguns exames complementares também poderem ser úteis nessa investigação, como a Radiografia, a Tomografia Computadorizada de Tórax, o Ecocardiografia transesofágica e o Eletrocardiograma, alguns pacientes não apresentam resultados alterados nesses exames, o que corrobora para a importância da história clínica no diagnóstico precoce e correto de DAA (Pape et al., 2015).

A dissecção pode ocorrer em qualquer segmento da artéria aorta e isso torna a DAA um processo dinâmico com uma gama ampla de sinais e sintomas (Gawinecka et al., 2017). Uma dor no peito ou nas costas, descrita como intensa, aguda, em facada, em rasgo ou "lacrimejante" e de início abrupto foi o sintoma mais encontrado em pacientes com essa emergência, sendo essa dor e suas características associadas positivamente com o diagnóstico de DAA (Gawinecka et al., 2017; Gorla et al., 2017; Nazerian, Giachino, et al., 2014). Segundo os estudos de (Mussa et al., 2016), a dor no peito foi relatada em 61,6\% dos casos, enquanto a dor nas costas foi relatada em 84,4\% deles. No Registro Internacional de Dissecção de Aorta, $85 \%$ dos pacientes apresentaram a dor torácica de início súbito (Elsayed et al., 2017). Ainda segundo os estudos de (Nazerian, Vanni, et al., 2014), 70,5\% dos pacientes apresentaram dor torácica, enquanto $25,6 \%$ deles apresentaram dor nas costas.

As evidências ainda apontam para uma diferença na prevalência da localização da dor de acordo com o tipo de DAA. De acordo com (Evangelista et al., 2018), a dor no peito foi descrita em $79 \%$ dos pacientes com DAA do tipo A, enquanto a mesma foi relatada em $63 \%$ dos pacientes com DAA do tipo B. Ainda segundo esse mesmo estudo, a dor nas costas foi mais comumente relatada em pacientes com DAA do tipo B (64\%), enquanto apenas $43 \%$ dos pacientes com DAA do tipo A apresentaram essa dor (Evangelista et al., 2018). Para reforçar esses achados, o estudo de Pape et al. (2015) traz que 85\% dos pacientes com DAA do tipo A queixaram-se de dor no peito, em comparação com $67 \%$ dos pacientes no tipo B, enquanto a dor nas costas foi relatada em $70 \%$ dos pacientes do tipo B e em $43 \%$ do tipo A.

Além da apresentação clássica de dor torácica ou na região dorsal, de início abrupto e bastante intensa, outros sinais e sintomas compõem o quadro clínico de DAA (Pape et al., 2015). De acordo com Nienaber et al. (2016), o desvio de fluxo de sangue para um falso lúmen que ocorre na DAA pode ocasionar sinais e sintomas de isquemia, como má perfusão, insuficiência da válvula aórtica e tamponamento. Segundo os estudos de Elsayed et al. (2017), em até 33\% dos pacientes com DAA do tipo A são relatados sintomas de má perfusão de órgão-alvo, sendo que a má perfusão cerebral, periférica e a visceral podem ocorrer separadas ou simultaneamente.

Ainda sobre a má perfusão e a DAA, de acordo com Patel et al. (2014), a má perfusão das artérias espinhais pode levar a sintomas neurológicos como paresia e paraplegia, enquanto a má perfusão das artérias viscerais pode causar mais frequentemente a dor abdominal. Além disso, o estudo de Jonker et al. (2015) mostrou que dor abdominal e dor nas pernas ocorrem mais comumente do que a dor no peito nos pacientes com DAA e com isquemia visceral. Também de acordo com Afifi et al. (2015), a dor abdominal (63\%) e nas pernas (25\%) ocorreram mais frequentemente nos pacientes com isquemia visceral. Segundo o estudo realizado com 281 pessoas de Nazerian, Vanni, et al. (2014), 14,2\% dos pacientes estudados apresentaram dor abdominal, $21 \%$ síncope e 7,5\% apresentaram sintomas de déficit de perfusão.

Quanto aos sintomas neurológicos, Nienaber e Clough (2015) dizem que inicialmente esses sintomas coexistem com a dor típica de DAA e podem variar entre sintomas nervosos centrais transitórios ou permanentes, incluindo síncope e vários sinais 
espinhais, como paraparesia ou paraplegia. De acordo com Pape et al. (2015), 19\% dos pacientes analisados com DAA do tipo A apresentaram síncope, enquanto apenas 3\% dos pacientes com DAA do tipo B apresentaram esse sintoma.

Outrossim, outros sintomas que são raros mas que podem estar associados à DAA são rouquidão, obstrução das vias aéreas superiores, ruptura na árvore traqueobrônquica com hemoptise, ruptura no esôfago com hematêmese, disfagia, síndrome da veia cava superior, massas cervicais pulsantes, síndrome de Horner ou a presença de um sopro contínuo (Elsayed et al., 2017).

Ao exame físico do paciente com DAA, alguns achados podem ser úteis no diagnóstico como a ausência de pulsos nos membros superiores e pulsos fracos ou não palpáveis nos membros inferiores, que sugerem o acometimento de diferentes artérias (Elsayed et al., 2017). Segundo os estudos de J et al. (2019), os déficits de pulso ao exame físico estavam presentes em 15,9\% dos pacientes diagnosticados com DAA, enquanto que, no estudo de Jonker et al. (2015), os déficits do pulso femoral estavam presentes em $32 \%$ dos pacientes com isquemia visceral. Outro conjunto de achados importantes é a Tríade de Beck, que pode indicar tamponamento cardíaco, a regurgitação aórtica aguda na presença de sopro diastólico ou B3 na ausculta cardíaca, hemotórax, além de perda unilateral dos sons respiratórios (Elsayed et al., 2017).

Durante a realização de exames complementares, especialmente os exames de imagem, alguns achados podem ser úteis no diagnóstico definitivo de DAA, como a identificação de um retalho íntimo e verdadeiro, assim como um lúmen falso na aorta (M et al., 2014). Ademais, outros achados de técnicas de imagem como derrame pericárdico, hematoma periaórtico e lúmen falso patente foram mais comuns em não sobreviventes de DAA, demonstrando que os resultados desses exames podem ser úteis na predição de um prognóstico, além fornecer um diagnóstico (Evangelista et al., 2018). De acordo com Pape et al. (2015), alterações nos exames de imagem estão presentes na maioria dos pacientes com DAA, sendo alguns achados importantes contorno aórtico anormal, mediastino superior aumentado e o "Sinal de cálcio".

Antes da realização de exames complementares, alguns estudos recomendam estimar o risco de DAA por meio de um escore que avalia 12 marcadores de risco, dentre eles, condições predisponentes, característica da dor e achados do exame físico (Gawinecka et al., 2017; Nazerian, Giachino, et al., 2014). Esse escore pode ser feito à beira leito e serve para a triagem dos pacientes e para classificá-los em risco baixo, moderado ou alto de terem DAA e, assim, orientar o profissional quanto à melhor abordagem e como deve-se seguir com a investigação diagnóstica (Gawinecka et al., 2017; Nazerian, Vanni, et al., 2014; Nazerian, Giachino, et al., 2014). Entretanto, segundo o estudo retrospectivo de Nazerian, Giachino, et al. (2014), essa ferramenta, apesar de razoavelmente sensível, deve ser utilizada com cautela por ser pouco específica e objetiva para estimar o risco de DAA.

Ainda segundo este mesmo estudo, devem ser realizados exames de imagem, o mais rápido possível, naqueles pacientes com alto risco de DAA e com risco intermediário sem diagnósticos alternativos (Nazerian, Giachino, et al., 2014). Além disso, o estudo de Nazerian, Giachino, et al. (2014) ainda sugere que, em todos os pacientes com risco baixo, a radiografia de tórax deve ser realizada, a fim de corroborar com o diagnóstico de DAA ou para descartá-la, firmando diagnósticos diferenciais.

De acordo com Gawinecka et al. (2017), os exames de imagem são importantes para diagnosticar a DAA, além de monitorar os pacientes com dilatação ou dissecção da artéria aorta. Atualmente, os principais e mais utilizados exames de imagem no diagnóstico de DAA são, entre outros, a Tomografia Computadorizada, Ressonância Magnética, Ecocardiografias transesofágica e transtorácica, a Radiografia de tórax e o Eletrocardiograma (Nienaber \& Clough, 2015; Pape et al., 2015; J et al., 2019). Entretanto, de acordo com a análise de Elsayed et al. (2017), os exames de rotina para diagnóstico de DAA como exames de sangue incluindo enzimas cardíacas para descartar infarto do miocárdio, eletrocardiograma e radiografia de tórax são raramente suficientes para confirmar o diagnóstico.

O uso da Tomografia Computadorizada (TC) para diagnóstico de DAA aumentou e se tornou o exame de imagem de primeira escolha, constituindo, juntamente com a Ressonância Magnética (RM), o padrão ouro para diagnóstico dessa emergência (Mussa et al., 2016; Gawinecka et al., 2017). De acordo com Gawinecka et al. (2017), a TC com contraste é o exame 
de imagem mais utilizado no diagnóstico de DAA, permitindo avaliar a extensão da dissecção, o tamanho do lúmen falso ou verdadeiro, envolvimentos de ramos arteriais, entre outros achados importantes. Segundo outro estudo, a TC sem contraste também é útil no diagnóstico de DAA e pode permitir a distinção entre lúmen falso de DAA trombosado e hematoma de ruptura de parede aneurismática (M et al., 2014).

Mais especificamente, a Angiografia por Tomografia Computadorizada (ATC) é, atualmente, a técnica diagnóstica de primeira escolha em casos de DAA, apresentando uma sensibilidade e especificidade que se aproximam de 100\% (Elsayed et al., 2017). Segundo alguns estudos, entre os benefícios desse exame de imagem estão a fácil execução e o fato de não ser invasivo, além de ser prontamente disponível, rápido e relativamente barato (Elsayed et al., 2017; Nienaber \& Clough, 2015). Ainda de acordo com o estudo de Elsayed et al. (2017), o diagnóstico de DAA do tipo A pode ser confirmado com esse exame a partir da visualização de dois lúmens diferentes na aorta ascendente separados por um retalho íntimo.

A ATC também pode ser útil para fornecer informações como a localização da laceração da íntima, a extensão da dissecção e o estado dos vasos em arco (Elsayed et al., 2017). Além disso, segundo os estudos de Jonker et al. (2015), esse exame também é importante nos casos de suspeita de DAA com isquemia visceral, pois, em cerca de $80 \%$ dos casos estudados, essa técnica de imagem mostrou o envolvimento dos vasos abdominais.

Entretanto, os estudos de Pare et al. (2016) trazem que, apesar de ser uma importante ferramenta diagnóstica de DAA, a realização da ATC em todos os pacientes que chegam ao departamento de emergência com dores características desse quadro seria inviável.

Como já citado, a RM, juntamente com a TC, é o padrão ouro para diagnóstico de DAA segundo os estudos de Mussa et al. (2016). Esse mesmo estudo ainda mostrou que a sensibilidade da RM foi de $95 \%$ a 100\%, enquanto sua especificidade foi de 94\% a 98\% (Mussa et al., 2016). Além disso, conforme descrito nas análises de Gawinecka et al. (2017), a RM é um método altamente adequado para o acompanhamento de pacientes e para a realização de exames seriados, pois permite a visualização de toda a artéria aorta, além de não expor o paciente a radiação ionizante ou contrates.

No entanto, essa ferramenta não é amplamente utilizada como técnica de diagnóstico inicial de DAA, apesar de ser bastante precisa (Gawinecka et al., 2017). Essa limitação da RM ocorre por ela ser um exame demorado, com pouca disponibilidade e dificuldade para monitorar o paciente durante a sua realização, além de ser incompatível com pacientes que possuem dispositivos metálicos implantados (Gawinecka et al., 2017; Elsayed et al., 2017).

Quanto ao papel da Ecocardiografia Transesofágica (ETE) na investigação diagnóstica de DAA, os autores Elsayed et al. (2017) trazem que é bastante útil devido à relação anatômica íntima do esôfago com a aorta. Dentre outros exames, a ETE deve ser um exame de escolha para o diagnóstico inicial em pacientes com baixo risco de dissecção aórtica (Gawinecka et al., 2017). Além disso, os mesmos autores sugerem que esse exame, dentre outros, também seja realizado naqueles pacientes com alto risco de DAA (Gawinecka et al., 2017).

De acordo com o estudo de Mussa et al. (2016), a sensibilidade desse exame foi entre $86 \%$ e 100\%, enquanto sua especificidade foi de $90 \%$ a $100 \%$. Nesse sentido, a ETE é vantajosa devido a sua capacidade de mostrar a presença de um lúmen falso ou verdadeiro e de prover informações sobre o funcionamento das válvulas ventricular e aórtica, além de ser uma opção de escolha nos casos em que o contraste está contraindicado (Elsayed et al., 2017).

Além disso, a ETE portátil pode ser bastante útil em condições emergenciais, nas quais o tempo é escasso e o paciente encontra-se hemodinamicamente instável (Gawinecka et al., 2017). Em contrapartida, os autores Gawinecka et al. (2017) analisaram que a interpretação desse exame depende da qualificação do profissional que o realiza e, assim, resultados falsopositivos ou falso-negativos podem ser comuns.

Este mesmo estudo de Gawinecka et al. (2017) ainda traz que a utilização dos exames TC, RM e ETE combinados para o diagnóstico de DAA tem uma sensibilidade de $98 \%$ a $100 \%$ e uma especificidade para as três técnicas de $95 \%$ a $98 \%$. 
Com relação à Ecocardiografia Transtorácica (ETT) no diagnóstico de DAA, os autores Gawinecka et al. (2017) sugerem que o principal benefício dessa técnica de imagem seja uma rápida e não invasiva avaliação de diversos segmentos da aorta, especialmente de sua raiz e da aorta ascendente proximal. Ademais, os autores descrevem sua rapidez e ampla disponibilidade, além de fornecer informações sobre o estado cardíaco e aórtico como pontos positivos desse exame (Gawinecka et al., 2017).

Entretanto, os mesmos autores elucidam sobre a limitação da sensibilidade diagnóstica da ETT, pois resultados negativos não seriam confiáveis para a exclusão da DAA (Gawinecka et al., 2017). Segundo os estudos de Mussa et al. (2016) demonstraram, a sensibilidade do exame foi de $73,3 \%$ a $100 \%$, com uma média de $86,9 \%$, enquanto a especificidade foi entre $71,2 \%$ e $91 \%$, com média de $81,1 \%$.

Outro exame de imagem que faz parte da investigação diagnóstica de DAA é a Radiografia de Tórax (RT), pelo fato da sua realização ser padrão nos pacientes que chegam ao pronto socorro com dor torácica (Evangelista et al., 2018). De acordo com Gawinecka et al. (2017), mediastino alargado ou contorno aórtico anormal na RT estão presentes em aproximadamente 50\% dos pacientes estudados. Entretanto, um resultado normal desse exame não é confiável para a exclusão do diagnóstico de DAA, já que, ainda segundo Gawinecka et al. (2017), 12\% dos pacientes não mostraram anormalidades, enquanto nos estudos de Evangelista et al. (2018), a porcentagem de paciente com DAA confirmada que não apresentavam anormalidades do mediastino ou contorno aórtico foi de mais de $20 \%$.

O Eletrocardiograma (ECG) de 12 derivações, assim como a RT, é um exame padrão para pacientes com dor torácica no departamento de emergência e, por isso, também faz parte da investigação de DAA (Evangelista et al., 2018). Esse exame pode ser útil em casos de suspeita de DAA, sendo a presença de alterações inespecíficas do segmento ST ou da onda T o achado mais frequente (Gawinecka et al., 2017). No entanto, um número significativo de $31 \%$ de pacientes do estudo de Gawinecka et al. (2017) não apresentou alterações no ECG, o que pode dificultar e atrasar o diagnóstico de DAA (Gawinecka et al., 2017; Evangelista et al., 2018).

De acordo com os estudos de Gawinecka et al. (2017), ambas a sensibilidade e a especificidade dos dois exames citados anteriormente, a RT e o ECG em conjunto, são bastante baixas para excluir ou confirmar o diagnóstico de DAA.

Outro exame complementar que também pode fazer parte do diagnóstico dessa emergência é o Dímero D (DD), um subproduto da trombose que funciona como ferramenta de estratificação de risco e biomarcador de DAA (Asha \& Miers, 2015; Gawinecka et al., 2017; Nazerian, Morello, et al., 2014). Conforme analisado por Nazerian, Morello, et al. (2014), o DD é relativamente barato e amplamente disponível, além de ter mostrado uma sensibilidade combinada de 97\% no diagnóstico de DAA.

Segundo alguns estudos, esse biomarcador tem alta sensibilidade negativa, sendo, assim, útil para descartar a DAA nas primeiras 24 horas após o início dos sintomas, levando em consideração o nível de corte de $500 \mathrm{ng} / \mathrm{ml}$, também utilizado para destacar embolia pulmonar (Asha \& Miers, 2015; Gawinecka et al., 2017; Evangelista et al., 2018). As análises ainda mostram que o DD é especialmente útil para destacar a DAA em pacientes com risco baixo para essa doença (Asha \& Miers, 2015; Gorla et al., 2017; Nazerian et al., 2018). Segundo Gorla et al. (2017), considerando aquelas pessoas que apresentaram baixo risco no escore de DAA, o DD apresentou uma sensibilidade de 93,5\% e uma especificidade de 63,2\%, com valor preditivo negativo de $98,9 \%$ e uma taxa de falha de $1,1 \%$.

Além do DD, discute-se o uso de outros biomarcadores no diagnóstico de DAA, como marcadores inflamatórios, proteínas de músculo liso, cadeia pesada de miosina e isoenzima de creatina quinase BB (Gawinecka et al., 2017; Evangelista et al., 2018). Apesar de serem considerados rápidos e econômicos, e terem mostrado uma possível utilidade no diagnóstico de DAA, esses biomarcadores ainda não estão disponíveis amplamente ou são usados frequentemente em pronto socorros (Asha \& Miers, 2015; Evangelista et al., 2018). 
Por fim, é importante citar a Ultrassonografia Cardíaca Focalizada (FOCUS) como uma técnica diagnóstica alternativa para DAA, que vem sendo cada vez mais utilizada nos departamentos de emergência para investigar pacientes com dor torácica (Nazerian, Vanni, et al., 2014; Pare et al., 2016). Ela é vantajosa por ser um exame que pode ser realizado em poucos minutos à beira leito, de forma segura e que está amplamente disponível, além de poder ser feito fora do ambiente hospitalar (Nazerian, Vanni, et al., 2014).

Segundo Nazerian, Vanni, et al. (2014), a FOCUS é útil para completar o exame físico do paciente, podendo detectar sinais ultrassonográficos diretos como retalho da íntima e hematoma intramural, além de sinais ultrassonográficos indiretos de DAA do tipo A, a exemplo de dilatação da aorta ascendente, tamponamento e insuficiência da válvula aórtica. Além disso, de acordo com os estudos de Pare et al. (2016), essa modalidade de exame pode ser utilizada para avaliar a aorta proximal em busca de aneurisma.

Algumas análises ainda mostraram que o diagnóstico de DAA foi mais rápido naqueles pacientes submetidos ao FOCUS, em detrimento dos que realizaram outra técnica diagnóstica, além de terem sido menos propensos a não receber um diagnóstico de DAA (Pare et al., 2016). De acordo com Nazerian, Vanni, et al. (2014), esse exame ainda pode ser útil como uma ferramenta de triagem, especialmente para pacientes com DAA do tipo A, identificando de forma rápida aqueles que precisam de exames avançados ou transferência para centros mais especializados. Ainda segundo esse mesmo estudo, entretanto, a FOCUS não é confiável para ser usada independentemente como um método diagnóstico de DAA do tipo A à beiro leito, mesmo quando combinada com o escore de risco (Nazerian, Vanni, et al., 2014).

\section{Complicações e consequências}

A incidência de DAA está relacionada com a idade, a média no início da dissecção é de 65 anos, sendo, em geral, os pacientes com dissecção do tipo A mais jovens que os com o tipo B (Wang et al., 2014). Homens possuem maior risco de desenvolvimento da dissecção dos dois tipos, além disso o tipo A ocorre com maior frequência que o B (Gawinecka et al., 2017). Ademais, no artigo de Dean et al. (2014) foi demonstrado que, devido a diversos determinantes psicossociais, pacientes afrodescendentes possuíram maior tendência de apresentar DAA relacionada ao uso de cocaína em idades menos avançadas em comparação a pacientes brancos, bem como maior tendência de apresentar HAS e diabetes mellitus.

A gravidade e a localização são variáveis que influenciam na seleção da melhor abordagem para reparo ou substituição da região danificada da aorta (Nienaber et al., 2016). O reparo cirúrgico aberto é o mais comum em casos de DAA ascendente e de arco aórtico (Nienaber et al., 2016; Nienaber \& Clough, 2015). Quando a dissecção ocorre na aorta descendente, a abordagem é clínica e, por vezes, ocorre a intervenção endovascular minimamente invasiva. Já em casos de complicação por má perfusão de órgão ou membro - dissecção progressiva - coleta de sangue extra aórtica, dor intratável, hipertensão não controlada ou expansão precoce do lúmen falso fazem-se necessárias abordagens individualizadas (Nienaber \& Clough, 2015).

As complicações da DAA englobam sinais de ruptura de aorta, envolvimento da artéria visceral, isquemia do membro inferior, diâmetro aórtico superior a $30 \mathrm{~mm}$, concomitante úlcera aórtica penetrante ou hematoma intramural e crescimento aórtico superior a $10 \mathrm{~mm} / \mathrm{ano}$ (J et al., 2019). Dentre as complicações com pior prognóstico, encontrou-se os pacientes com histórico de troca valvar aórtica, dor torácica migratória, choque, tamponamento e isquemia pré-operatória (Elsayed et al., 2017). No estudo de Wc et al. (2019), uma meta-análise de dados de bases eletrônicas, a taxa de mortalidade foi de 30,8\% para pacientes com isquemia visceral e 9,1\% para aqueles sem isquemia visceral. A isquemia visceral aguda, onde ocorre diminuição do fluxo de sangue para as artérias celíacas e/ou mesentéricas é uma das complicações mais letais da DAA (Jonker et al., 2015).

A dissecção da aorta ascendente apresenta alta mortalidade, entre $26 \%$ e $58 \%$, sendo a proximidade da válvula aórtica e grandes vasos um fator que potencializa as chances de complicações (Mussa et al., 2016). Esses pacientes têm sua taxa de mortalidade influenciada por condições pré-operatórias, entre elas, troca valvar aórtica prévia, dor torácica migratória, isquemia 
de membro pré-operatório, hipotensão durante a apresentação ou choque/tamponamento cardíaco. O comprometimento renal antes da operação, a baixa fração de ejeção do ventrículo esquerdo e a idade também são fatores que influenciam na mortalidade a longo prazo (Gawinecka et al., 2017).

\section{Fatores de risco, precauções e tratamento}

No estudo de Evangelista et al. (2018), cobriu-se as conclusões críticas do instituto de radiologia IRAD, a arteriosclerose esteve presente em $27 \%$ dos pacientes, aneurisma aórtico conhecido previamente em 16\%, síndrome de Marfan em 5\%, causas iatrogênicas em $4 \%$ e o uso de cocaína em 1,8\% dos pacientes. Nesse mesmo estudo, observou-se como diferencial que a síndrome de Marfan foi mais comum em pacientes menores de 40 anos, enquanto a hipertensão arterial sistêmica (HAS) nesses pacientes foi menos comum (Evangelista et al., 2018). No entanto, a HAS mostrou-se o fator de risco mais importante da DAA, uma vez que cerca de $80 \%$ de todos pacientes com DAA a possuem (Gawinecka et al., 2017). A hipertensão arterial além de aumentar a pressão na parede da aorta, agredindo a camada íntima, é capaz de atuar na degradação da matriz extracelular pela elevação de moléculas pró-inflamatórias e metaloproteinases, o que pode acarretar na dissecção aórtica (Gawinecka et al., 2017; Nienaber et al., 2016).

O tabagismo é outro fator de risco para DAA, uma vez que, em comparação aos não fumantes, pacientes tabagistas podem ter dissecção aórtica e aneurisma de aorta com uma frequência duas vezes maior (Gawinecka et al., 2017). Outros fatores de risco incluem: dislipidemia, idade avançada, traumas, uso de drogas (cocaína e anfetaminas), inflamação vascular, doença autoimune, doença infecciosa, aneurisma de aorta e fatores iatrogênicos. Em relação aos fatores genéticos, algumas condições predispõem a dissecção aórtica, dentre elas: Síndrome de Marfan, Síndrome de Ehlers-Danlos, Síndrome de Loeys-Dietz e a Síndrome de Turner (Elsayed et al., 2017; Nienaber et al., 2016). Sexo masculino também é um fator de risco para essa doença, uma vez que a DAA é mais prevalente em homens (Melvinsdottir et al., 2016).

O tratamento clínico inicial da dissecção aórtica depende do local da laceração e da evidência de complicações e visa o manejo dos fatores de risco, através do controle da pressão arterial, por exemplo. O objetivo é limitar a propagação da dissecção, pela redução do estresse na parede aórtica. Além disso, se busca a redução da pressão de pulso, a fim de manter a perfusão do órgão alvo, para esse fim, o tratamento de primeira linha é o uso de fármacos $\beta$-bloqueadores (Nienaber \& Clough, 2015). A aplicação de protocolos padronizados é uma importante medida para reduzir o tempo de diagnóstico e orientar os profissionais sobre o manejo clínico inicial e o tratamento adequado a cada paciente, o que possibilita uma otimização do processo desde o diagnóstico até a intervenção cirúrgica (Gawinecka et al., 2017).

O acompanhamento de pacientes após a dissecção é uma importante medida na prevenção de complicações, pois $38 \%$ dos pacientes com dissecção aórtica tipo B manejados clinicamente apresentam complicações posteriores e precisam de intervenção (Patel et al., 2014), sendo o maior risco apresentado nos primeiros 2 anos após a dissecção (Nienaber et al., 2016). Dessa forma, a realização de exames de imagem periódicos com CT ou RM da aorta torácica permanece como terapia ideal Patel et al. (2014), e ajudam na avaliação da evolução dilatação da aorta (Gawinecka et al., 2017). Esse procedimento deve ser realizado após a alta do tratamento endovascular ou aberto (Nienaber \& Clough, 2015).

Exames de imagem oferecem uma base ideal para o diagnóstico e acompanhamento da dilatação da aorta, porém alguns biomarcadores têm apresentado potencial para a estratificação de risco de pacientes com dissecção aórtica. O nível de PCR, por exemplo, é um marcador útil para pacientes com DAA do tipo A na escolha de tratamento e percepção de risco (M et al., 2014). Marcadores laboratoriais como a elevação de enzimas hepáticas e pancreáticas podem ser indicativos de complicação de dissecção aórtica aguda tipo B (Gawinecka et al., 2017).

A escolha da melhor abordagem na dissecção aórtica depende de alguns fatores como: a gravidade, a localização e os riscos de complicações. O reparo cirúrgico aberto rápido é mais usado na dissecção aguda tipo A envolvendo a aorta ascendente 
e o arco aórtico, embora abordagens híbridas possam ser uma escolha (Nienaber et al., 2016; Nienaber \& Clough, 2015). Estudos apontam o reparo endovascular (RE) usando stents sendo benéfico também em casos de dissecções de aorta proximal e de arco aórtico (Elsayed et al., 2017).

A melhor escolha de tratamento para dissecções do tipo B ainda está sendo elucidada, necessitando de uma estratificação de risco para direcionar o manejo clínico (Patel et al., 2014). O reparo endovascular profilático em pacientes com dissecção aórtica aguda do tipo B sem complicações não demonstrou ser a escolha mais benéfica a curto prazo, nesses casos, o tratamento clínico é o mais indicado (Tolenaar et al., 2014). Para os casos complicados de dissecção tipo B, como a dissecção associada à isquemia visceral, o tratamento precoce é essencial para minimizar o tempo de isquemia, já que apresenta alto risco de mortalidade (Jonker et al., 2015) e o reparo endovascular é uma importante ferramenta de tratamento (Afifi et al., 2015).

Além disso, o acompanhamento de pacientes que receberam tratamento com reparo endovascular necessita de melhores estratégias. Em um estudo comparando a sobrevida de 5 anos em pacientes com DA tipo B observou que pacientes que receberam terapia com RE apresentaram maior propensão a apresentar doença complicada. Porém, em uma comparação com a mortalidade, o grupo tratado com RE apresentou resultados significativamente menores (Patel et al., 2014). Em comparação com o reparo aberto da aorta o reparo endovascular da aorta torácica teve melhores resultados (Afifi et al., 2015).

Atualmente, para tratamento de dissecção de aorta abdominal isolada (DAAI) tem sido preferível o reparo endovascular em detrimento da cirurgia aberta. Porém, ainda não há um consenso sobre o tratamento ideal para um caso de DAAI, sendo a escolha do melhor tratamento baseada nos sintomas e nas complicações, buscando primeiramente o tratamento menos invasivo. O tratamento conservador com avaliação contínua deve ser considerado para pacientes sintomáticos, porém se os sintomas persistirem a abordagem invasiva deve ser realizada ( $\mathrm{J}$ et al., 2019).

\section{Conclusão}

Baseando-se nos aspectos observados e discutidos anteriormente, através da metodologia proposta foi possível a análise e compreensão dos artigos selecionados de modo a apresentar boas e eficientes estratégias de diagnóstico precoce da dissecção aguda aórtica. Além disso, o presente estudo também permitiu o entendimento patológico da DAA, bem como de boas formas de abordagem e de tratamento.

De modo geral, a bibliografia utilizada apresentou uma excelente base teórica acerca da patologia em si, como também das principais complicações, das duas consequências, dos métodos de diagnóstico, dos fatores de risco, das precauções necessárias e do tratamento dos diversos tipos de dissecção aórtica. Com isso, este artigo poderá servir de fundamentação teórica aos profissionais da saúde no tratamento da doença de dissecção aórtica.

Ademais, a análise dos artigos também permitiu identificar lacunas no conhecimento que merecem maior pesquisa, como o desenvolvimento de melhores formas de tratamento da doença. Devido à gravidade e importância da DAA, torna-se necessário a produção de mais artigos e de estudos a respeito desse tema, principalmente no que se diz respeito às melhores estratégias de tratamento.

Por fim, o presente artigo apresentou os dados necessários sobre as melhores formas de identificar e de diagnosticar precocemente um caso de dissecção aguda de aorta em um paciente com queixa de dor torácica no departamento de emergência. Com isso, torna-se mais provável que os acadêmicos e profissionais consigam garantir aos pacientes portadores de DAA melhores prognósticos, evitando possíveis sequelas e mortes.

Para trabalhos futuros, sugerimos que sejam realizadas pesquisas mais aprofundadas acerca das melhores formas de tratamento para casos de dissecções aórticas do tipo B. Além disso, achamos pertinente a realização de estudos futuros que estabeleçam melhores formas de acompanhamento de pacientes que receberam tratamento com reparo endovascular. 


\section{Referências}

Afifi, R., Sandhu, H., Leake, S., Boutrous, M., \& ... (2015). Outcomes of patients with acute type B (DeBakey III) aortic dissection: A 13-year, single-center experience. Circulation, Query date: 2020-10-17 14:10:14. https://www.ahajournals.org/doi/abs/10.1161/circulationaha.115.015302

Albuquerque, L. C., Braile, D. M., Palma, J. H., Saadi, E. K., Almeida, R. M. S. de, Gomes, W. J., \& Buffolo, E. (2009). Diretrizes para o tratamento cirúrgico das doenças da aorta da Sociedade Brasileira de Cirurgia Cardiovascular: Atualização 2009. Revista Brasileira de Cirurgia Cardiovascular, 24(2), 7-33. https://doi.org/10.1590/S0102-76382009000300004

Asha, S., \& Miers, J. (2015). A systematic review and meta-analysis of D-dimer as a rule-out test for suspected acute aortic dissection. Annals of emergency medicine, Query date: 2020-10-17 14:10:14. https://www.sciencedirect.com/science/article/pii/S0196064415001183

Chang, Y.-J., Chang, S.-L., Chong, E., Suenari, K., \& Michalopoulos, A. (2017). Cardiovascular Emergencies. BioMed Research International, 2017, 1-2. https://doi.org/10.1155/2017/7210261

Dean, J., Woznicki, E., O’Gara, P., \& ... (2014). Cocaine-related aortic dissection: Lessons from the International Registry of Acute Aortic Dissection. The American journal of ..., Query date: 2020-10-17 14:10:14. https://www.sciencedirect.com/science/article/pii/S000293431400391X

Elsayed, R., Cohen, R., Fleischman, F., \& ... (2017). Acute type A aortic dissection. Cardiology ..., Query date: 2020-10-17 14:10:14. https://www.cardiology.theclinics.com/article/S0733-8651(17)30023-1/abstract

Evangelista, A., Isselbacher, E., Bossone, E., \& ... (2018). Insights from the International Registry of Acute Aortic Dissection: A 20-year experience of collaborative clinical research. Circulation, Query date: 2020-10-17 14:10:14. https://www.ahajournals.org/doi/abs/10.1161/circulationaha.117.031264

Gawinecka, J., Schönrath, F., \& Eckardstein, A. von. (2017). Acute aortic dissection: Pathogenesis, risk factors and diagnosis. Swiss medical weekly, Query date: 2020-10-17 14:10:14. https://www.zora.uzh.ch/id/eprint/148742/

Gorla, R., Erbel, R., Kahlert, P., Tsagakis, K., \& ... (2017). Accuracy of a diagnostic strategy combining aortic dissection detection risk score and D-dimer levels in patients with suspected acute aortic syndrome. European Heart ..., Query date: 2020-10-17 14:10:14. https://journals.sagepub.com/doi/abs/10.1177/2048872615594497

Hsia, R., Hale, Z., \& Tabas, J. (2016). A national study of the prevalence of life-threatening diagnoses in patients with chest pain. JAMA internal medicine, Query date: 2020-10-17 14:10:14. https://jamanetwork.com/journals/jamainternalmedicine/article-abstract/2527387

J, W., M, Z., J, Q., Y, H., Y, C., C, Y., \& Ja, E. (2019, dezembro). A systematic review and meta-analysis of isolated abdominal aortic dissection. Journal of Vascular Surgery; J Vasc Surg. https://doi.org/10.1016/j.jvs.2019.04.467

Jonker, F., Patel, H., Upchurch, G., \& ... (2015). Acute type B aortic dissection complicated by visceral ischemia. The Journal of Thoracic ..., Query date: 202010-17 14:10:14. https://www.sciencedirect.com/science/article/pii/S0022522314017887

M, K., K, O., D, U., K, Y., T, S., H, S., A, M., H, A., Y, O., \& M, I. (2014). Diagnostic utility of unenhanced computed tomography for acute aortic syndrome. Circulation Journal : Official Journal of the Japanese Circulation Society; Circ J. https://doi.org/10.1253/circj.cj-14-0198

Melvinsdottir, I., Lund, S., Agnarsson, B., \& ... (2016). The incidence and mortality of acute thoracic aortic dissection: Results from a whole nation study. European Journal of ..., Query date: 2020-10-17 14:10:14. https://academic.oup.com/ejcts/article-abstract/50/6/1111/2726579

Mussa, F., Horton, J., Moridzadeh, R., Nicholson, J., \& ... (2016). Acute aortic dissection and intramural hematoma: A systematic review. Jama, Query date: 2020-10-17 14:10:14. https://jamanetwork.com/journals/jama/article-abstract/2544646

Nazerian, P., Giachino, F., Vanni, S., \& ... (2014). Diagnostic performance of the aortic dissection detection risk score in patients with suspected acute aortic dissection. European Heart ..., Query date: 2020-10-17 14:10:14. https://journals.sagepub.com/doi/abs/10.1177/2048872614527010

Nazerian, P., Morello, F., Vanni, S., Bono, A., \& ... (2014). Combined use of aortic dissection detection risk score and D-dimer in the diagnostic workup of suspected acute aortic dissection. International journal of ... https://www.sciencedirect.com/science/article/pii/S016752731400953X

Nazerian, P., Mueller, C., Soeiro, A., Leidel, B., \& ... (2018). Diagnostic accuracy of the aortic dissection detection risk score plus D-dimer for acute aortic syndromes: The ADvISED Prospective Multicenter Study. Circulation, Query date: 2020-10-17 14:10:14. https://www.ahajournals.org/doi/abs/10.1161/CIRCULATIONAHA.117.029457

Nazerian, P., Vanni, S., Castelli, M., Morello, F., \& ... (2014). Diagnostic performance of emergency transthoracic focus cardiac ultrasound in suspected acute type A aortic dissection. Internal and emergency ..., Query date: 2020-10-17 14:10:14. https://link.springer.com/content/pdf/10.1007/s11739-014-1080-9.pdf

Nienaber, C., \& Clough, R. (2015). Management of acute aortic dissection. The Lancet, Query date: 2020-10-17 14:10:14. https://www.sciencedirect.com/science/article/pii/S0140673614610059

Nienaber, C., Clough, R., Sakalihasan, N., \& ... (2016). Aortic dissection. Nature Reviews ..., Query date: 2020-10-17 14:10:14. https://www.nature.com/articles/nrdp201653?platformlu003doscarlu0026draftlu003dcollection

Pape, L., Awais, M., Woznicki, E., Suzuki, T., \& ... (2015). Presentation, diagnosis, and outcomes of acute aortic dissection: 17-year trends from the International Registry of Acute Aortic Dissection. Journal of the ..., Query date: 2020-10-17 14:10:14. https://www.onlinejacc.org/content/66/4/350.abstract

Pare, J., Liu, R., Moore, C., Sherban, T., \& ... (2016). Emergency physician focused cardiac ultrasound improves diagnosis of ascending aortic dissection. The American Journal of ..., Query date: 2020-10-17 14:10:14. https://www.sciencedirect.com/science/article/pii/S073567571501061X 
Research, Society and Development, v. 10, n. 2, e28110212542, 2021

(CC BY 4.0) | ISSN 2525-3409 | DOI: http://dx.doi.org/10.33448/rsd-v10i2.12542

Patel, A., Eagle, K., \& Vaishnava, P. (2014). Acute type B aortic dissection: Insights from the International Registry of Acute Aortic Dissection. Annals of cardiothoracic surgery, Query date: 2020-10-17 14:10:14. https://www.ncbi.nlm.nih.gov/pmc/articles/PMC4128929/

Pereira, A. S., Shitsuka, D. M., Parreira, F. J., \& Shitsuka, R. (2018). Metodologia da pesquisa científica. [e-book]. Santa Maria/RS. Ed. UAB/NTE/UFSM. Recuperado de: https://repositorio.ufsm.br/bitstream/handle/1/15824/Lic_Computacao_Metodologia-Pesquisa-Cientifica.pdf?sequence=1.

Tolenaar, J., Froehlich, W., Jonker, F., \& ... (2014). Predicting in-hospital mortality in acute type B aortic dissection: Evidence from International Registry of Acute Aortic Dissection. Circulation, Query date: 2020-10-17 14:10:14. https://www.ahajournals.org/doi/abs/10.1161/CIRCULATIONAHA.113.007117

Wang, W., Duan, W., Xue, Y., Wang, L., Liu, J., Yu, S., \& ... (2014). Clinical features of acute aortic dissection from the Registry of Aortic Dissection in China. The Journal of thoracic ..., Query date: 2020-10-17 14:10:14. https://www.sciencedirect.com/science/article/pii/S0022522314010411

Wc, H., Bm, H., Cc, H., P, M., M, O., \& J, L. (2019, outubro). Prognostic Role of Admission C-Reactive Protein Level as a Predictor of In-Hospital Mortality in Type-A Acute Aortic Dissection: A Meta-Analysis. Vascular and Endovascular Surgery; Vasc Endovascular Surg. https://doi.org/10.1177/1538574419858161 